Tendências em Matemática Aplicada e Computacional, 3, No. 1 (2002), 61-70.

(C) Uma Publicação da Sociedade Brasileira de Matemática Aplicada e Computacional.

\title{
Generalizing the Real Interval Arithmetic
}

R. CALLEJAS-BEDREGAL, Departamento de Matemática, Universidade Federal da Paraíba, 58059-900 João Pessoa, PB, Brasil

B.R.C. BEDREGAL, R.H.N. SANTIAGO, Laboratório de Lógica e Inteligência Computacional, Departamento de Informática e Matemática Aplicada, Universidade Federal do Rio Grande do Norte, 59072-970 Natal, RN, Brasil.

\begin{abstract}
In this work we propose a generalized real interval arithmetic. Since the real interval arithmetic is constructed from the real arithmetic, it is reasonable to extend it to intervals on any domain which has some algebraic structure, such as field, ring or group structure. This extension is based on the local equality theory of Santiago $[11,12]$ and on an interval constructor which mappes bistrongly consistently complete dcpos into bifinitely consistently complete dcpos.
\end{abstract}

\section{Introduction}

R. Moore [10] developed an interval mathematic in order to proporcionate the control of errors in numeric computations in the solution of problems concerning real numbers. Nevertheless, the real interval arithmetic of Moore is not completely faithful with the real arithmetic, in the sense that the algebraic structure of the interval arithmetic is not a field.

Several works have been developed to overcome the algebraic incompatibility between the set of intervals and the set of real numbers [8]. In [11] was observed that the problem does not come from the algebraic structure of intervals but from the adopted primitive notion of equality for intervals. Thus, Santiago proposed a new notion of equality for intervals, called local equality, and states some of the consequences of such approach on intervals, like the possible simulation of a field structure on intervals. He presented a domain theoretic approach for intervals and Scott's simple equality; he restricted the last to local equality and showed that intervals can be used like real numbers.

The Moore theory not only comprehend real intervals, but also complex intervals, matrix and array of real and complex numbers. Actually, several programming languages have been developed using this interval data type as primitive, this class of programming languages are denominated XSC (eXtension for Scientific Computation) [7, 15]. It is reasonable to hope that in the future will be developed XSC languages with a parametric data type. Thus, in order to provide a theoretical foundation to this class of programming languages, it is necessary to generalize the interval theory in such a way that include the above kind of intervals and any other 
possible type of intervals, i.e. that consider the parametric data type. It means that we have to see intervals as a constructor. Since real intervals are defined through a partial order on the real set, we might define intervals on any partially order set. In [6] was associated to every poset their set of intervals. The work of [5] uses the set of intervals of a poset with a coherent relation as primitive object (a web) of a coherent space. Thus, they do not use it as a categorical interval constructor on the poset category. In $[2,3,4]$ this construction was worked out from a categorical sight: it was treated as a categorical constructor. In this sense were studied some categories of domains which are closed under this constructor and where the projection functions $l: \mathbb{I}(\mathbf{D}) \longrightarrow \mathbf{D}$ and $r: \mathbb{I}(\mathbf{D}) \longrightarrow \mathbf{D}$ defined by $l([a, b])=a$ and $r([a, b])=b$, respectively, are morphisms of these categories.

In this work, we propose a generalized real interval arithmetic based on this interval constructor and the local equality theory of Santiago [11, 12]. We show how to extend the interval arithmetic (on the real numbers) to other domains obtained via this interval constructor.

\section{Partial Ordered Sets}

Let $P$ be a set. A binary relation $\leq$ on $P$ is said a partial order on $P$ if for each $x, y, z \in P$ the following conditions are satisfied:

Reflexivity: $x \leq x$;

Antisymmetry: if $x \leq y$ and $y \leq x$ then $x=y$;

Transitity if $x \leq y$ and $y \leq z$ then $x \leq z$.

A set $P$ with a partial order $\leq$ on $P$ is said a partial ordered set or simplely a poset.

Let $\mathbf{D}=\langle D, \leq\rangle$ be a poset. A non empty set $\Delta \subseteq D$ is called directed if $\forall a, b \in \Delta \exists c \in \Delta$ such that $a \leq c$ and $b \leq c$. A poset $\mathbf{D}$ is directed complete (dcpo for short) if each directed set $\Delta$ has a least upper bound or supremum (denoted by $\sqcup \Delta$ ) and a cpo if in addition it has a least element.

Let $\mathbf{D}$ and $\mathbf{E}$ be dcpo's. A function $f: E \longrightarrow D$ is called continuous w.r.t. the orders or simplely continuous if it is monotonic $(x \leq y$ implies $f(x) \leq f(y))$ and preserves least upper bounds of directed sets $(f(\bigsqcup \Delta)=\bigsqcup f(\Delta))$.

\section{The Interval Constructor}

Scott in [13] defined an information order on $\mathbb{I}(\mathbb{R})=\{[r, s] / r, s \in \mathbb{R}$ and $r \leq s\}$ :

$$
[a, b] \sqsubseteq[c, d] \Leftrightarrow a \leq c \leq d \leq b
$$

Posteriously, Acióly in [1], used this order as a base to develop a computational foundation to the Moore Interval Theory. 
Notice that both the real intervals and the order on the set of real intervals depends upon the usual real order. Thus, we can generalize this constructions by considering, instead of the real set with its usual order, any partially ordered set, it means that we can think of intervals as a constructor on the category POSET.

Definition 1. Let $\mathbf{D}=\langle D, \leq\rangle$ be a poset. The poset $\mathbb{I}(\mathbf{D})=\langle\mathbb{I}(D)$, $\rangle$, where

- $\mathbb{I}(D)=\{[a, b] / a, b \in D$ and $a \leq b\}$

- $[a, b] \sqsubseteq[c, d] \Leftrightarrow a \leq c$ and $d \leq b$

is called the poset of intervals of $\mathbf{D}$.

There are two natural functions from $\mathbb{I}(D)$ to $D$, which are the left and right projections $l: \mathbb{I}(D) \longrightarrow D$ and $r: \mathbb{I}(D) \longrightarrow D$ respectively, defined by

$$
l([a, b])=a \text { and } r([a, b])=b
$$

Clearly the function $l$ is monotonic and therefore it is a morphism from the poset $\mathbb{I}(\mathbf{D})$ to the poset $\mathbf{D}$, but the function $r$ is not monotonic. But this could be overcome by reverting the order on $\mathbf{D}$.

Definition 2. Let $\mathbf{D}=\langle D, \leq\rangle$ be a poset. The reverse poset of $\mathbf{D}$, denoted by $\mathbf{D}^{o p}$, is the pair $\mathbf{D}^{o p}=\left\langle D^{o p}, \leq_{o p}\right\rangle$, where $D^{o p}=D$ and $x \leq_{o p} y$ if $y \leq x$.

Clearly every poset $\mathbf{D}$ has a reverse poset and the function $r: \mathbb{I}(D) \longrightarrow D^{o p}$ is monotonic.

Notice that not every reverse poset of a dcpo is a dcpo. This motivate the following definition:

Definition 3. Let $\mathbf{D}=\langle D, \leq\rangle$ be a dcpo. $\mathbf{D}$ is called reversable if $\mathbf{D}^{o p}$ is a dcpo.

Proposition 1. Let $\mathbf{D}=\langle D, \leq\rangle$ be a reversable dcpo. Then $\mathbb{I}(\mathbf{D})=\langle\mathbb{I}(D)$, $\rangle$ is a reversable dcpo and the projections $l: \mathbb{I}(D) \longrightarrow D$ and $r: \mathbb{I}(D) \longrightarrow D^{\text {op }}$ are continuous.

Proposition 2. Let $\mathbf{D}_{1}=\left\langle D_{1}, \leq_{1}\right\rangle$ and $\mathbf{D}_{2}=\left\langle D_{2}, \leq_{2}\right\rangle$ be reversable dcpos. Then $\mathbf{D}_{1} \times \mathbf{D}_{2}$ is a reversable dcpo and $\mathbb{I}\left(\mathbf{D}_{1} \times \mathbf{D}_{2}\right) \cong \mathbb{I}\left(\mathbf{D}_{1}\right) \times \mathbb{I}\left(\mathbf{D}_{2}\right)$.

Proposition 3. Let $\mathbf{D}_{1}=\left\langle D_{1}, \leq_{1}\right\rangle$ and $\mathbf{D}_{2}=\left\langle D_{2}, \leq_{2}\right\rangle$ be reversable dcpos. Let $f, g: D_{1} \longrightarrow D_{2}$ be continuous functions such that $f \sqsubseteq g$ (i.e., $f(x) \leq_{2} g(x)$ for each $\left.x \in D_{1}\right)$. Then the function $F: \mathbb{I}\left(D_{1}\right) \longrightarrow \mathbb{I}\left(D_{2}\right)$ defined by $F([a, b])=[f(a), g(b)]$ is the unique continuous function which makes the following diagrams: 


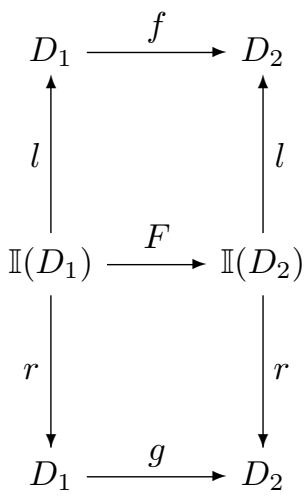

commutative.

The above proposition generalizes the notion of interval extension of a real function.

Definition 4. A dcpo $\mathbf{D}=\langle D, \leq\rangle$ is consistently complete if for each $S \subseteq D$ non empty such that $S$ has an upper bound then it has a supreme $\bigsqcup S$ in $\mathbf{D}$. If $\mathbf{D}$ and $\mathbf{D}^{o p}$ are consistently complete dcpos then $\mathbf{D}$ is said biconsistently complete.

Proposition 4. Let $\mathbf{D}=\langle D, \leq\rangle$ be a biconsistently complete dcpo. Then $\mathbb{I}(\mathbf{D})$ is biconsistently complete dcpo.

Definition 5. A consistently complete dcpo $\mathbf{D}=\langle D, \leq\rangle$ is strongly consistently complete if each $S \subseteq D$ finite and non empty has an upper bound. If $\mathbf{D}$ and $\mathbf{D}^{o p}$ are strongly consistently complete dcpos then $\mathbf{D}$ is said bistrongly consistently complete

The interval constructor $\mathbb{I}$ is not closed on bistrongly consistently complete dcpos. But, we have

Corollary 1. Let $\mathbf{D}=\langle D, \leq\rangle$ be a bistrongly consistently complete dcpo. Then $\mathbb{I}(\mathbf{D})$ is biconsistently complete dcpo.

Proof: Since each bistrongly consistently complete dcpo is a biconsistently complete dcpo, the corollary is straightforward from proposition 4 .

\section{Local Equality}

Let $A$ be a set and $\{0,1\}$ the standard boolean set. The standard equality relation "=" on $A$ is modeled by the boolean function $\llbracket .=. \rrbracket: A \times A \rightarrow\{0,1\}$, where the following axioms are satisfied:

1. $x=x$ (reflexivity); 
2. $x=y \Rightarrow y=x$ (symmetry);

3. $x=y \wedge y=z \Rightarrow x=z$ (transitivity).

In [14], Scott proposed an alternative axiomatization for equality aiming to capture partial defined objects. This axiomatization replaces the axiom of reflexivity for

$$
\text { (refl) } x=x \Leftrightarrow E x
$$

This axiom is based on the fact that when we are dealing with partial defined objects the law of reflexivity does not make sense in a formalized theory. For example, $\frac{1}{a}=\frac{1}{a}$ does not make sense in all rings. This axiom reads off "if we can say that $x$ is equal to itself, then we can say that it exists and if $x$ exists then we can say that it is equal to itself' - e.g. $\frac{1}{a}=\frac{1}{a} \Leftrightarrow E \frac{1}{a}$. The standard theory of equality is derived from this one [14].

Santiago extended Scott's simple equality in such a way that consistency will become a weaker equivalence relation [11].

We say that the elements $a$ and $b$ in a poset $\mathbf{D}=\langle D, \leq\rangle$ are consistent, denoted by $a \bigodot b$, if there exist an element $c \in D$ such that $a \leq c$ and $b \leq c$. Consistency satisfies the axioms of reflexivity and refl since $x \smile x$, and the axiom of symmetry since $x \frown y \Rightarrow y \frown x$. However, it does not satisfies the axiom of transitivity. To overcome this situation, we define a precondition for the transitivity axiom, giving rise to the theory of local equality.

Definition 6 (Theory of Local Equality). The axioms for local equality are:

1. $x \stackrel{\text { Loc }}{=} x \Leftrightarrow \operatorname{Ex}($ refl);

2. $x \stackrel{\text { Loc }}{=} y \Rightarrow y \stackrel{\text { Loc }}{=} x$ (symmetry);

3. $E(x \vee z) \Rightarrow(x \stackrel{\text { Loc }}{=} y \wedge y \stackrel{\text { Loc }}{=} z \rightarrow x \stackrel{\text { Loc }}{=} z)$. (Local transitivity).

So, in this theory we restrict the application of transitivity of Scott's simple equality.

Notice that if $\stackrel{L o c}{=}$ is a local equality on a set $S$ and $x=y$ for some $x, y \in S$, then, by property (refl) ( $x$ and $y$ are the same elements!!), $x \stackrel{\text { Loc }}{=} y$.

\section{Ordered Local Algebras}

It is possible to generalize the notion of classical algebraic structures, such as group, ring and field considering a local equality instead of the usual equality.

Definition 7. Let $\stackrel{\text { Loc }}{=}$ be a local equality on the set $D$ and two binary operations, + and $*$ on D. Consider the following statements: 
1. $a+(b+c) \stackrel{\text { Loc }}{=}(a+b)+c$ for each $a, b, c \in D$;

2. There exist an unique element $0 \in D$ such that for each a $\in D$, $a+0 \stackrel{\text { Loc }}{=} a \stackrel{\text { Loc }}{=} 0+a$

3. For each $a \in D$, there exist an element $(-a) \in D$ such that $a+(-a) \stackrel{\text { Loc }}{=} 0 \stackrel{\text { Loc }}{=}$ $(-a)+a$ and for any $b \in D$ such that $a+b \stackrel{\text { Loc }}{=} 0 \stackrel{\text { Loc }}{=} b+a, b \stackrel{\text { Loc }}{=}-a$;

4. $a+b \stackrel{\text { Loc }}{=} b+a$ for each $a, b \in D$;

5. $a *(b+c) \stackrel{\text { Loc }}{=}(a * b)+(a * c)$ for each $a, b, c \in D$;

6. $a *(b * c) \stackrel{\text { Loc }}{=}(a * b) * c$ for each $a, b, c \in D$;

7. There exist an unique element $1 \in D$ such that for each a $\in D$, $a * 1 \stackrel{\text { Loc }}{=} a \stackrel{\text { Loc }}{=} 1 * a$;

8. $a * b \stackrel{\text { Loc }}{=} b * a$ for each $a, b \in D$;

9. For each $a \in D$, a not locally equal to 0 there exist an element $a^{-1} \in D$ such that $a * a^{-1} \stackrel{\text { Loc }}{=} 1 \stackrel{\text { Loc }}{=} a^{-1} * a$ and for any $b \in D$ such that $a * b \stackrel{\text { Loc }}{=} 1 \stackrel{\text { Loc }}{=} b * a$, $b \stackrel{\text { Loc }}{=} a^{-1}$.

The structure $\langle D, \stackrel{L o c}{=},+\rangle$ is a local group if it satisfies 1,2 and 3. It is a local abelian group if it is a local group and satisfies 4 . The structure $\langle D, \stackrel{\text { Loc }}{=},+, *\rangle$ is a local ring if $\langle D, \stackrel{L o c}{=},+\rangle$ is a local abelian group and satisfies 5 and 6 . It is a local commutative ring if it is a local ring and satisfies 8. It is a local commutative ring with unity if it is a local comutative ring and satisfies $\%$. It is a local field if it satisfies all the statements.

In this definition, the unicity conditions are with respect to the local equality.

Definition 8. $\mathbf{D}=\langle D, \leq, \stackrel{L o c}{=},+\rangle$ is said an ordered local group (resp. ordered local abelian group) if

1. $\langle D, \leq\rangle$ is a poset;

2. $\langle D, \stackrel{\text { Loc }}{=},+\rangle$ is a local group (resp. local abelian group);

3. the + is motonic w.r.t. $\leq$, i.e. if $a \leq c$ and $b \leq d$ then $a+b \leq c+d$.

Definition 9. $\mathbf{D}=\langle D, \leq, \stackrel{L o c}{=},+, *\rangle$ is said an ordered local ring (resp. commutative ring, commutative ring with unity, field) if

1. $\langle D, \leq\rangle$ is a poset; 
2. $\langle D, \stackrel{\text { Loc }}{=},+, *\rangle$ is a local ring (resp. commutative ring, commutative ring with unity, field);

3. the + is motonic w.r.t. $\leq$, i.e. if $a \leq c$ and $b \leq d$ then $a+b \leq c+d$;

4. Given $a \in D$, a satisfies either:

(i) $\forall x, y \in D$, such that $x \leq y, a^{\prime} * x \leq a^{\prime} * y$ and $x * a^{\prime} \leq y * a^{\prime} \forall a^{\prime} \in D$ such that $a \leq a^{\prime}$

or

(ii) $\forall x, y \in D$, such that $x \leq y, a^{\prime} * y \leq a^{\prime} * x$ and $y * a^{\prime} \leq x * a^{\prime} \forall a^{\prime} \in D$ such that $a^{\prime} \leq a$.

If the local equality is an equality, we drop the word local from the above definitions

Lemma 1. Let $\mathbf{D}=\langle D, \leq,=,+, *\rangle$ be a ring such that $\langle D, \leq\rangle$ is a bistrongly consistently complete dcpo. Define on $\mathbb{I}(\mathbf{D})$ the following operations:

$$
\begin{gathered}
{[a, b] \oplus[c, d]=[a+c, b+d]} \\
{[a, b] \otimes[c, d]=\left[\prod A, \bigsqcup A\right]}
\end{gathered}
$$

where $A=\{a * c, a * d, b * c, b * d\}$. Then $\oplus$ and $\otimes$ are well defined.

Proof: Since the sum in $D$ is monotonic w.r.t $\leq$, we have that $\forall[a, b],[c, d] \in \mathbb{I}(D),[a, b] \oplus[c, d]=[a+c, b+d] \in \mathbb{I}(D)$. Since $(D, \leq)$ is a bistrongly consistently complete dcpo, we have that $\prod A, \sqcup A \in D$ and $\Pi A \leq \bigsqcup A$, where $A=\{a * c, a * d, b * c, b * d\}$. Thus, $\left[\prod A, \bigsqcup A\right] \in \mathbb{I}(D)$

Theorem 1. Let $\mathbf{D}=\langle D, \leq,=,+, *\rangle$ be an ordered ring (resp. commutative ring, commutative ring with unity, field) such that $\langle D, \leq\rangle$ is a bistrongly consistently complete dcpo. Then $\mathbb{I}(\mathbf{D})=\langle\mathbb{I}(D), \sqsubseteq \stackrel{\text { Loc }}{=}, \oplus, \otimes\rangle$; where

$[a, b] \stackrel{\text { Loc }}{=}[c, d]$ iff $E([a, b] \sqcup[c, d])$, is an ordered local ring (resp. commutative ring with unity, field).

Proof: Suppose that $\langle D, \leq,=,+, *\rangle$ is an ordered ring with $(D, \leq)$ a bistrongly consistently complete dcpo. We must prove that $\langle\mathbb{I}(D), \sqsubseteq \stackrel{\text { Loc }}{=}, \oplus, \otimes\rangle$ is an ordered local ring. It is straightforward to prove that $\langle\mathbb{I}(D), \sqsubseteq \stackrel{\text { Loc }}{=}, \oplus\rangle$ is an ordered local abelian group with $\mathbf{0}=[-\mathbf{a}, \mathbf{a}]$ as the identity element of $\mathbb{I}(D)$.

In order to prove property 5 (of Definition 7 ) for $\mathbb{I}(D)$, let $\left[a, a^{\prime}\right],\left[b, b^{\prime}\right],\left[c, c^{\prime}\right] \in$ $\mathbb{I}(D)$. By definition, we have that

$$
\left[a, a^{\prime}\right] \otimes\left(\left[b, b^{\prime}\right] \oplus\left[c, c^{\prime}\right]\right)=\left\lceil\prod A, \bigsqcup A\right]
$$

where $A=\left\{a * b+a * c, a * b^{\prime}+a * c^{\prime}, a^{\prime} * b+a^{\prime} * c, a^{\prime} * b^{\prime}+a^{\prime} * c^{\prime}\right\}$. On the other hand, we have that 


$$
\left(\left[a, a^{\prime}\right] \otimes\left[b, b^{\prime}\right]\right) \oplus\left(\left[a, a^{\prime}\right] \otimes\left[c, c^{\prime}\right]\right)=\left[\Pi C+\prod D, \bigsqcup C+\bigsqcup D\right]
$$

where $C=\left\{a * b, a * b^{\prime}, a^{\prime} * b, a^{\prime} * b^{\prime}\right\}$ and $D=\left\{a * c, a * c^{\prime}, a^{\prime} * c, a^{\prime} * c^{\prime}\right\}$. Notice that, by the monotonocity of the sum in $D$, we have that

$$
\Pi_{C}+\Pi_{D} \leq \Pi_{A} \leq \bigsqcup A \leq \bigsqcup C+\bigsqcup D .
$$

Thus, $\left[\prod_{C}+\prod D, \sqcup C+\sqcup D\right] \sqsubseteq\left[\prod A, \sqcup A\right]$. Therefore, $[\lceil C+\lceil D, \sqcup C+\sqcup D] \stackrel{\text { Loc }}{=}$ $\lceil A, \sqcup A]$, which prove property 5 . $\mathbb{I}(D)$.

In order to prove property 6 (of Definition 7 ) for $\mathbb{I}(D)$, let $\left[a, a^{\prime}\right],\left[b, b^{\prime}\right],\left[c, c^{\prime}\right] \in$

By definition, we have that $\left[b, b^{\prime}\right] \otimes\left[c, c^{\prime}\right]=[x, y]$ where $x=\prod\left\{b * c, b * c^{\prime}, b^{\prime} *\right.$ $\left.c, b^{\prime} * c^{\prime}\right\}$ and $y=\bigsqcup\left\{b * c, b * c^{\prime}, b^{\prime} * c, b^{\prime} * c^{\prime}\right\}$ Also,

$$
\left[a, a^{\prime}\right] \otimes\left(\left[b, b^{\prime}\right] \otimes\left[c, c^{\prime}\right]\right)=\left[\prod A, \bigsqcup A\right]
$$

where $A=\left\{a * x, a * y, a^{\prime} * x, a^{\prime} * y\right\}$. Analogously, we have that $\left[a, a^{\prime}\right] \otimes\left[b, b^{\prime}\right]=[z, w]$ where $z=\prod\left\{a * b, a * b^{\prime}, a^{\prime} * b, a^{\prime} * b^{\prime}\right\}$ and $y=\bigsqcup\left\{a * b, a * b^{\prime}, a^{\prime} * b, a^{\prime} * b^{\prime}\right\}$ Also,

$$
\left(\left[a, a^{\prime}\right] \otimes\left[b, b^{\prime}\right]\right) \otimes\left[c, c^{\prime}\right]=\left\lceil\prod B, \bigsqcup B\right]
$$

where $B=\left\{z * c, z * c^{\prime}, w * c, w * c^{\prime}\right\}$.

Let $C=\left\{a * b * c, a * b * c^{\prime}, a * b^{\prime} * c, a * b^{\prime} * c^{\prime}, a^{\prime} * b * c, a^{\prime} * b * c^{\prime}, a^{\prime} * b^{\prime} * c, a^{\prime} * b^{\prime} * c^{\prime}\right\}$. There are several cases we must analize. We will only prove property 6 in one special case, the other cases been analogous. So, we suppose that $a$ and $c$ satisfy property 4 (i) of Definition 9. In this case, we will prove that

$$
\Pi A \leq \Pi_{C} \leq \bigsqcup C \leq \bigsqcup A .
$$

In fact, since $x=\prod\left\{b * c, b * c^{\prime}, b^{\prime} * c, b^{\prime} * c^{\prime}\right\}$ we have that

$$
a * x \leq \prod\left\{a * b * c, a * b * c^{\prime}, a * b^{\prime} * c, a * b^{\prime} * c^{\prime}\right\} .
$$

On the other hand, since $a \leq a^{\prime}$ and $a$ satisfies property $4(i)$, we have that

$$
a^{\prime} * x \leq \prod\left\{a^{\prime} * b * c, a^{\prime} * b * c^{\prime}, a^{\prime} * b^{\prime} * c, a^{\prime} * b^{\prime} * c^{\prime}\right\} .
$$

Therefore,

$$
\prod A \leq\left(a * x \sqcap a^{\prime} * x\right) \leq \prod C .
$$

Analogously we prove that $\bigsqcup C \leq \bigsqcup A$. Since

$$
\Pi A \leq \Pi C \leq \bigsqcup C \leq \bigsqcup A
$$

we have that $[\Pi A, \sqcup A] \sqsubseteq\left[\lceil C, \sqcup C]\right.$. Analogously, we can prove that $\left[\prod B, \sqcup B\right] \sqsubseteq$ $[\lceil C, \sqcup C]$. Therefore, 


$$
\left[\prod A, \bigsqcup A\right] \stackrel{\text { Loc }}{=}\left[\prod B, \bigsqcup B\right]
$$

as we wanted. The remaining statements of the theorem are easy to prove.

Notice that:

1. If $[a, b]$ and $[c, d]$ are degenerates intervals (i.e. $a=b$ and $c=d$ ), then $[a, b] \oplus[c, d]$ and $[a, b] \otimes[c, d]$ are the degenerates intervals $[a+c, a+c]$ and $[a * c, a * c]$, respectively.

2. If $x \in[a, b]$ (i.e. $a \leq x \leq b$ ) and $y \in[c, d]$ then $x+y \in[a, b] \oplus[c, d]$ and $x * y \in[a, b] \otimes[c, d]$.

3. If $\mathbf{D}=\langle\mathbb{R}, \leq,=, *,+\rangle$ where $\leq$ is the usual order, the local equality is the usual equality $=$ and + and $*$ are addition and product on real numbers, then $\langle\mathbb{I}(\mathbb{R}), \oplus, \otimes\rangle$ is the Moore interval arithmetic.

\section{Final Remarks}

In spite that the parametric interval data type is not actually part of any programming language, this work provide a theoretical foundations of the possibility to construct programming languages which consider a parametric interval data type as primitive in such a way that the arithmetics operators can be implemented using polymorphism. It is clear, from this work, that the algebraic properties of this arithmetic operators depend upon the algebraic properties of the respective operators in the parametrized data type.

Resumo Neste trabalho propomos uma aritmética real intervalar generalizada. Como a aritmética intervalar real é construída da aritmética real, é razoável estender esta para intervalos de qualquer domínio que tenha alguma estrutura algébrica, tais como estruturas de corpo, anel ou grupo. Esta extensão é baseada sobre a teoria da igualdade local de Santiago $[11,12]$ e sobre um construtor que mapeia dcpos bifortemente consistentemente completos em dcpos bifinitamente consistentemente completos.

\section{References}

[1] B.M. Acióly, "Uma Fundamentação Computacional da Matemática Intervalar", Ph.D. thesis, CPGCC da UFRGS, Porto Alegre, RS, 1991.

[2] R. Callejas-Bedregal and B. R. Callejas Bedregal, Intervals as a domain constructor, in "Seletas do XXIII CNMAC", Tendências em Matemática Aplicada e Computacional, 2 (2001), 43-52.

[3] R. Callejas-Bedregal and B. R. Callejas Bedregal, BiScott dcpos as cartesian closed category, in "Pre-proceeding of the Second International Symposium on Domain Theory, Chengdu-China, pp. 22-26, october, 2001. 
[4] R. Callejas-Bedregal and B. R. Callejas Bedregal, Interval as a categorical constructor, in "IV Workshop on Formal Methods, Rio de Janeiro, 01-02 October, pages 139-150, 2001.

[5] G.P. Dimuro, A.C.R. Costa and D.M. Claudio. A bi-structured coherence space for a global representation of the system IR of real intervals, in "CIT'99: Trends in Information Technology", (Proceedings of the International Conference on Information Technology, Bubhaneswar, December 20-22, 1999), Tata McGrawHill, pp. 173-178, 2000.

[6] G. Grätzer, "General Lattice Theory", Academic Press, New York, 1978.

[7] R.B. Kearfott, "Rigorous Global Search: Continuous Problems", Kluwer Academic Publishers, Dordrecht, 1996.

[8] S.M. Markov. On the extended interval arithmetic, Computes Rendus de L'Acadèmie Bulgare des Sciences, 2, No. 31 (1978), 163-166.

[9] R.E. Moore, "Interval Analysis", Prentice Hall, New Jersey, 1966.

[10] R.E. Moore, "Methods and Applications for Interval Analysis", SIAM, Philadelphia.

[11] R.H.N. Santiago, "The Theory of Interval Local Equations", Ph.D.thesis, Federal University of Pernambuco, PE, Brazil, 1999.

[12] R.H.N. Santiago, Interval local theory: toward a model for real type, in "IV Workshop on Formal Methods", Rio de Janeiro, 01-02 October, pp. 108-114, 2001.

[13] D.S. Scott, Outline of a mathematical theory of computation, in "4 ${ }^{\text {th }}$ Annual Princeton Conference on Information Sciences and Systems", p. 169-176, 1970.

[14] D.S. Scott, Identity and existency in intuitionistic logic, in Fourman, M. et al., editors, in "Lecture Notes in Mathematics" (Fourman, M. et al., eds.), 753, pp 660-696, Springer-Verlag, Durham, 1977.

[15] XSC-Languages, http://www.math.uni-wuppertal.de/org/WRST/xsc-sprachen. html, Access in may of 2002. 